\title{
Study of Baryon Acoustic Oscillations with SDSS DR13 Data and Measurements of $\Omega_{k}$ and
} $\Omega_{\mathrm{DE}}(\boldsymbol{a})$

\author{
B. Hoeneisen \\ Universidad San Francisco de Quito, Quito, Ecuador \\ Email:bruce1@fnal.gov, bhoeneisen@usfq.edu.ec
}

How to cite this paper: Hoeneisen, B. (2017) Study of Baryon Acoustic Oscillations with SDSS DR13 Data and Measurements of $\Omega_{k}$ and $\Omega_{D E}(a)$. International Journal of Astronomy and Astrophysics, 7, 11-27.

https://doi.org/10.4236/ijaa.2017.71002

Received: December 14, 2016

Accepted: February 4, 2017

Published: February 7, 2017

Copyright $\odot 2017$ by author and Scientific Research Publishing Inc. This work is licensed under the Creative Commons Attribution International License (CC BY 4.0).

http://creativecommons.org/licenses/by/4.0/

\begin{abstract}
We measure the baryon acoustic oscillation (BAO) observables $\hat{d}_{\alpha}\left(z, z_{c}\right)$, $\hat{d}_{z}\left(z, z_{c}\right)$, and $\hat{d}_{/}\left(z, z_{c}\right)$ as a function of red shift $z$ in the range 0.1 to 0.7 with Sloan Digital Sky Survey (SDSS) data release DR13. These observables are independent and satisfy a consistency relation that provides discrimination against miss-fits due to background fluctuations. From these measurements and the correlation angle $\theta_{\mathrm{MC}}$ of fluctuations of the Cosmic Microwave Background (CMB), we obtain $\Omega_{k}=-0.015 \pm 0.030$, $\Omega_{\mathrm{DE}}+2.2 \Omega_{k}=0.717 \pm 0.004$ and $w_{1}=0.37 \pm 0.61$ for dark energy density allowed to vary as $\Omega_{\mathrm{DE}}(a)=\Omega_{\mathrm{DE}}\left[1+w_{1}(1-a)\right]$. We present measurements of $\Omega_{\mathrm{DE}}(a)$ at six values of the expansion parameter $a$. Fits with several scenarios and data sets are presented. The data is consistent with space curvature parameter $\Omega_{k}=0$ and $\Omega_{\mathrm{DE}}(a)$ constant.
\end{abstract}

\section{Keywords}

Cosmology, Dark Energy, Baryon Acoustic Oscillations

\section{Introduction}

Peaks in the density of the primordial universe are the sources of acoustic waves of the tightly coupled plasma of photons, electrons, protons and helium nuclei. These acoustic waves propagate a distance $r_{S}^{\prime} \approx 145 \mathrm{Mpc}$ until the time of recombination and decoupling $t_{\mathrm{dec}}$ [1] [2] (All distances in this article are co-moving, i.e. are referred to the present time $t_{0}$ ). The baryon acoustic oscillation (BAO) distance $r_{S}^{\prime}$ corresponds to the observed correlation angle $\theta_{\mathrm{MC}}$ of fluctuations of the cosmic microwave background (CMB) [2]. Dark 
matter follows the BAO waves. The results, well after decoupling, for an initial point-like peak in the density, are two concentric shells of overdensity of radius $\approx 145 \mathrm{Mpc}$ and $\approx 18 \mathrm{Mpc}$ [1] [3] [4]. The inner spherical shell becomes reprocessed by the hierarchical formation of galaxies [5], while the outer shell is unprocessed to better than $1 \%$ [4] [6] (or even $0.1 \%$ with corrections [4] [6]) and therefore is an excellent standard ruler to measure the expansion parameter $a(t)$ of the universe as a function of time $t$. Histograms of galaxy-galaxy distances show an excess in the approximate range 145-11 Mpc to $145+11$ Mpc. We denote by $d_{\mathrm{BAO}}^{\prime}$ the mean of this BAO signal. We set $r_{S}^{\prime}=d_{\mathrm{BAO}}^{\prime} f$, where $f$ is a correction factor due to the peculiar motions of galaxies ( $f$ depends on the orientation of the galaxy pair with respect to the line of sight). Measurements of these BAO signals are well established: see References [3] and [4] for extensive lists of early publications.

In this article, we present studies of BAO with Sloan Digital Sky Survey (SDSS) publicly released data DR13 [7]. The study has three parts:

1) We measure the BAO observables $\hat{d}_{\alpha}\left(z, z_{c}\right), \hat{d}_{z}\left(z, z_{c}\right)$, and $\hat{d}_{l}\left(z, z_{c}\right)$ [8] in six bins of redshift $z$ from 0.1 to 0.7 . These observables are galaxygalaxy correlation distances, in units of $c / H_{0}$, of galaxy pairs respectively transverse to the line of sight, along the line of sight, and in an interval of angles with respect to the line of sight, for a reference (fictitious) cosmology.

2) We measure the space curvature parameter $\Omega_{k}$ and the dark energy density relative to the critical density $\Omega_{\mathrm{DE}}(a)$ as a function of the expansion parameter $a$ with the following BAO data used as an uncalibrated standard ruler: $\hat{d}_{\alpha}\left(z, z_{c}\right), \hat{d}_{z}\left(z, z_{c}\right)$, and $\hat{d}_{/}\left(z, z_{c}\right)$ for $0.1<z<0.7$ (this analysis), $\theta_{\mathrm{MC}}$ for $z_{\mathrm{dec}}=1089.9 \pm 0.4$ from Planck satellite observations [2] [9], and measurements of BAO distances in the Lyman-alpha ( $\operatorname{Ly} \alpha$ ) forest with SDSS BOSS DR11 data at $z=2.36 \quad[10]$ and $z=2.34$ [11].

3) Finally, we use the BAO measurements as a calibrated standard ruler to constrain a wider set of cosmological parameters.

The present analysis with DR13 data [7] closely follows the methods developed in References [8] and [12] for data release DR12 [13].

\section{BA0 Observables}

To define the quantities being measured we write the (generalized) Friedmann equation that describes the expansion history of a homogeneous universe:

$$
\frac{1}{H_{0}} \frac{1}{a} \frac{\mathrm{d} a}{\mathrm{~d} t} \equiv E(a)=\sqrt{\frac{\Omega_{\mathrm{m}}}{a^{3}}+\frac{\Omega_{r}}{a^{4}}+\Omega_{\mathrm{DE}}(a)+\frac{\Omega_{k}}{a^{2}}} .
$$

The expansion parameter $a(t)$ is normalized so that $a\left(t_{0}\right)=1$ at the present time $t_{0}$. The Hubble parameter $H_{0} \equiv 100 \mathrm{~h} \mathrm{~km} \cdot \mathrm{s}^{-1} \cdot \mathrm{Mpc}^{-1}$ is normalized so that $E(1)=1$ at the present time, i.e.

$$
\Omega_{\mathrm{m}}+\Omega_{\mathrm{r}}+\Omega_{\mathrm{DE}}(1)+\Omega_{k}=1 .
$$

The terms under the square root in Equation (1) are densities relative to the critical density of, respectively, non-relativistic matter, ultra-relativistic radiation, 
dark energy (whatever it is), and space curvature. In the General Theory of Relativity $\Omega_{\mathrm{DE}}(a)$ is constant. Here, we allow $\Omega_{\mathrm{DE}}(a)$ be a function of $a$ to be determined by observations. Measuring $\Omega_{k}$ and $\Omega_{\mathrm{DE}}(a)$ is equivalent to measuring the expansion history of the universe $a(t)$. The expansion parameter $a$ is related to redshift $z$ by $a=1 /(1+z)$.

The distance $d^{\prime}$ between two galaxies observed with a relative angle $\alpha$ and redshifts $Z_{1}$ and $Z_{2}$ can be written, with sufficient accuracy for our purposes, as [8].

$$
\begin{gathered}
d^{\prime} \equiv \frac{c}{H_{0}} d, \\
d=\sqrt{d_{\alpha}^{2}+d_{z}^{2}}, \\
d_{\alpha}=2 \sin \left(\frac{\alpha}{2}\right) \sqrt{\chi\left(z_{1}\right) \chi\left(z_{2}\right)}\left[1+\frac{1}{6} \Omega_{k} \chi\left(z_{1}\right) \chi\left(z_{2}\right)\right], \\
d_{z}=\chi\left(z_{1}\right)-\chi\left(z_{2}\right), \quad \text { where } \\
\chi(z) \equiv \int_{0}^{z} \frac{\mathrm{d} z^{\prime}}{E\left(z^{\prime}\right)} .
\end{gathered}
$$

$d_{\alpha}$ and $d_{z}$ are the distance components, in units of $c / H_{0}$, transverse to the line of sight and along the line of sight, respectively. $(\chi(z)$ should not be confused with the $\chi^{2}$ of fits). The difference between the approximation (3) and the exact expression for $d^{\prime}$, given by Equation (3.19) of Reference [14], is negligible for two galaxies at the distance $d_{\mathrm{BAO}}$ : the term of $d_{\alpha}$ proportional to $\Omega_{k}$ in Equation (3) changes by $0.1 \%$ at $z=0.7$.

We find the following approximations to $\chi(z)$ and $1 / E(z)$ valid in the range $0 \leq z<1$ with precision approximately $\pm 1 \%$ for $z_{c} \approx 3.79$ [8]:

$$
\chi(z) \approx z \exp \left(-\frac{z}{z_{c}}\right), \quad \frac{1}{E(z)} \approx\left(1-\frac{z}{z_{c}}\right) \exp \left(-\frac{z}{z_{c}}\right) .
$$

Our strategy is as follows. We consider galaxies with redshift in a given range $z_{\min }<z<z_{\max }$. For each galaxy pair we calculate $d_{\alpha}\left(z, z_{c}\right), d_{z}\left(z, z_{c}\right)$ and $d\left(z, z_{c}\right)$ with Equations (3) with the approximation (4) and fill one of three histograms of $d\left(z, z_{c}\right)$ (with weights to be discussed later) depending on the ratio $d_{z}\left(z, z_{c}\right) / d_{\alpha}\left(z, z_{c}\right)$ :

- If $d_{z}\left(z, z_{c}\right) / d_{\alpha}\left(z, z_{c}\right)<1 / 3$ fill a histogram of $d\left(z, z_{c}\right)$ that obtains a BAO signal centered at $\hat{d}_{\alpha}\left(z, z_{c}\right)$. For this histogram, $d_{z}^{2}\left(z, z_{c}\right)$ is a small correction relative to $d_{\alpha}^{2}\left(z, z_{c}\right)$ that is calculated with sufficient accuracy with the approximation (4), i.e. an error less than $0.2 \%$ on $\hat{d}_{\alpha}\left(z, z_{c}\right)$.

- If $d_{\alpha}\left(z, z_{c}\right) / d_{z}\left(z, z_{c}\right)<1 / 3$ fill a second histogram of $d\left(z, z_{c}\right)$ that obtains a BAO signal centered at $\hat{d}_{z}\left(z, z_{c}\right)$. For this histogram, $d_{\alpha}^{2}\left(z, z_{c}\right)$ is a small correction relative to $d_{z}^{2}\left(z, z_{c}\right)$ that is calculated with sufficient accuracy with the approximation (4) and $\Omega_{k}=0$, i.e. an error less than $0.2 \%$ on $\hat{d}_{z}\left(z, z_{c}\right)$.

- Else, fill a third histogram of $d\left(z, z_{c}\right)$ that obtains a BAO signal centered at $\hat{d}_{l}\left(z, z_{c}\right)$. 
Note that these three histograms have different galaxy pairs, i.e. have independent signals and independent backgrounds.

The galaxy-galaxy correlation distance $d_{\mathrm{BAO}}$, in units of $c / H_{0}$, is obtained from the BAO observables $\hat{d}_{\alpha}\left(z, z_{c}\right), \hat{d}_{z}\left(z, z_{c}\right)$, or $\hat{d}_{/}\left(z, z_{c}\right)$ as follows:

$$
\begin{gathered}
d_{\mathrm{BAO}}=\hat{d}_{\alpha}\left(z, z_{c}\right) \frac{\chi(z)\left[1+\frac{1}{6} \Omega_{k} \chi^{2}(z)\right]}{z \exp \left(-z / z_{c}\right)}, \\
d_{\mathrm{BAO}}=\hat{d}_{z}\left(z, z_{c}\right) \frac{1}{\left(1-z / z_{c}\right) \exp \left(-z / z_{c}\right) E(z)}, \\
d_{\mathrm{BAO}}=\hat{d}_{/}\left(z, z_{c}\right)\left(\frac{\chi(z)\left[1+\frac{1}{6} \Omega_{k} \chi^{2}(z)\right]}{z \exp \left(-z / z_{c}\right)}\right)^{n / 3}\left(\frac{1}{\left(1-z / z_{c}\right) \exp \left(-z / z_{c}\right) E(z)}\right)^{1-n / 3} .
\end{gathered}
$$

A numerical analysis obtains $n=1.70$ for $z=0.2$, dropping to $n=1.66$ for $z=0.8 \quad$ (in agreement with the method introduced in Reference [1] that obtains $n \approx 2$ when $d$ covers all angles). The redshift $z$ in Equations (5), (6) and (7) corresponds to the weighted mean of $z$ in the interval $z_{\min }$ to $z_{\max }$. The fractions in Equations (5), (6) and (7) are within $\approx 1 \%$ of 1 for $z_{c}=3.79$. Note that the limits of $\hat{d}_{\alpha}\left(z, z_{c}\right)$ or $\hat{d}_{z}\left(z, z_{c}\right)$ or $\hat{d}_{/}\left(z, z_{c}\right)$ as $z \rightarrow 0$ are all equal to $d_{\mathrm{BAO}}$.

The independent BAO observables $\hat{d}_{\alpha}\left(z, z_{c}\right), \hat{d}_{z}\left(z, z_{c}\right)$, and $\hat{d}_{l}\left(z, z_{c}\right)$ satisfy the consistency relation

$$
Q \equiv \frac{\hat{d}_{/}\left(z, z_{c}\right)}{\hat{d}_{\alpha}\left(z, z_{c}\right)^{n / 3} \hat{d}_{z}\left(z, z_{c}\right)^{1-n / 3}}=1 .
$$

The approximations in Equations (4) obtain galaxy-galaxy correlation distances $\hat{d}_{\alpha}\left(z, z_{c}\right), \hat{d}_{z}\left(z, z_{c}\right)$, and $\hat{d}_{/}\left(z, z_{c}\right)$ of a reference (fictitious) cosmology. We emphasize that these approximations are undone by Equations (5), (6), and (7) so in the end $d_{\mathrm{BAO}}$ has the correct dependence on the cosmological parameters which is different for Equations (5), (6), and (7).

The BAO observables $\hat{d}_{\alpha}\left(z, z_{c}\right), \hat{d}_{z}\left(z, z_{c}\right)$, and $\hat{d}_{/}\left(z, z_{c}\right)$ were chosen because 1) they are dimensionless, 2 ) they are independent, 3 ) they do not depend on any cosmological parameter, 4) they are almost independent of $Z$ (for an optimized value of $z_{c} \approx 3.79$ ) so that a large bin $z_{\max }-z_{\min }$ may be analyzed, and 5) satisfy the consistency relation (8) which allows discrimination against fits that converge on background fluctuations instead of the $\mathrm{BAO}$ signal.

It is observed that fluctuations in the CMB have a correlation angle [2] [9].

$$
\theta_{\mathrm{MC}}=0.010410 \pm 0.000005 \text {, }
$$

(we have chosen a measurement by the Planck collaboration with no input from $\mathrm{BAO})$. The extreme precision with which $\theta_{\mathrm{MC}}$ is measured makes it one of the primary parameters of cosmology. The correlation distance $r_{S}$, in units of $c / H_{0}$, is obtained from $\theta_{\mathrm{MC}}$ as follows: 


$$
r_{\mathrm{S}}=2 \sin \left(\frac{1}{2} \theta_{\mathrm{MC}}\right) \chi\left(z_{\mathrm{dec}}\right)\left[1+\frac{1}{6} \Omega_{k} \chi^{2}\left(z_{\mathrm{dec}}\right)+\frac{1}{120} \Omega_{k}^{2} \chi^{4}\left(z_{\mathrm{dec}}\right)\right] .
$$

For $\chi\left(z_{\text {dec }}\right)$ we do not neglect $\Omega_{r} \equiv \Omega_{\gamma} N_{\text {eq }} / 2$ of photons or neutrinos (we take $N_{\text {eq }}=3.38$ [2] corresponding to 3 neutrino flavors).

\section{Galaxy Selection and Data Analysis}

The present analysis is based on publicly released SDSS-IV DR13 data described in Reference [7], and includes the SDSS-III Baryon Oscillation Spectroscopic Survey (BOSS) [15], and the SDSS-IV Extended Baryon Oscillation Spectroscopic Survey (eBOSS) [16] which are designed for BAO measurements. A list of participating institutions in the SDSS-IV is given in the acknowledgment.

We obtain the following data from the SDSS DR13 catalog [7] for all objects identified as galaxies that pass quality selection flags: right ascension ra, declination dec, redshift $z$, redshift uncertainty $z E r r$, and the absolute value of the magnitude $r$. We require a good measurement of redshift, i.e.

$z E r r<0.001$. The present study is limited to galaxies with right ascension in the range $110^{\circ}$ to $270^{\circ}$, declination in the range $-5^{0}$ to $70^{\circ}$, and redshift in the range 0.1 to 0.7 . The galactic plane divides this data set into two independent sub-sets: the northern galactic cap $(\mathrm{N})$ and the southern galactic cap (S) defined by dec $\gtrless 27.0^{\circ}-17.0^{\circ}\left[\left(\mathrm{ra}-185.0^{\circ}\right) /\left(260.0^{\circ}-185.0^{\circ}\right)\right]^{2}$.

We calculate the absolute luminosity $F$ of galaxies relative to the absolute luminosity of a galaxy with $r=19.0$ at $z=0.35$, and calculate the corresponding magnitude $r_{35}$. We consider galaxies with $17.0<r_{35}<23.0$ (G). We define "luminous galaxies" (LG) with, for example, $r_{35}<19.2$, and "clusters" (C). Clusters $\mathrm{C}$ are based on a cluster finding algorithm that starts with LG's as seeds, calculates the total absolute luminosity of all G's within a distance 0.006 (in units of $c / H_{0}$ ), and then selects local maximums of these total absolute luminosities above a threshold, e.g. $r_{35}<16.6$.

A "run" is defined by a range of redshifts $\left(z_{\min }, z_{\max }\right)$, a data set, and a definition of galaxy and "center". For each of 6 bins of redshift $z$ from 0.10 to 0.70 , and each of 5 offset bins of $z$ from 0.15 to 0.65 , and for each data set $N$ or S, and for each choice of galaxy-center G-G, G-LG, LG-LG, or G-C (with several absolute luminosity cuts), we fill histograms of galaxy-center distances $d\left(z, z_{c}\right)$ and obtain the BAO distances $\hat{d}_{\alpha}\left(z, z_{c}\right), \hat{d}_{z}\left(z, z_{c}\right)$, and $\hat{d}_{l}\left(z, z_{c}\right)$ by fitting these histograms.

Histograms are filled with weights $(0.033 / d)^{2}$ or $F_{i} F_{j}(0.033 / d)^{2}$, where $F_{i}$ and $F_{j}$ are the absolute luminosities $F$ of galaxy $i$ and center $j$ respectively. We obtain histograms with $z_{c}=3.79,3$ and 5 . The reason for this large degree of redundancy is the difficulty to discriminate the BAO signal from the background with its statistical fluctuations and cosmological fluctuations due to galaxy clustering. Pattern recognition is aided by multiple histograms with different background fluctuations, and by the characteristic shape of the BAO signal that has a lower edge at approximately 0.031 and an upper edge at approximately 0.036 as shown in Figure 1. 

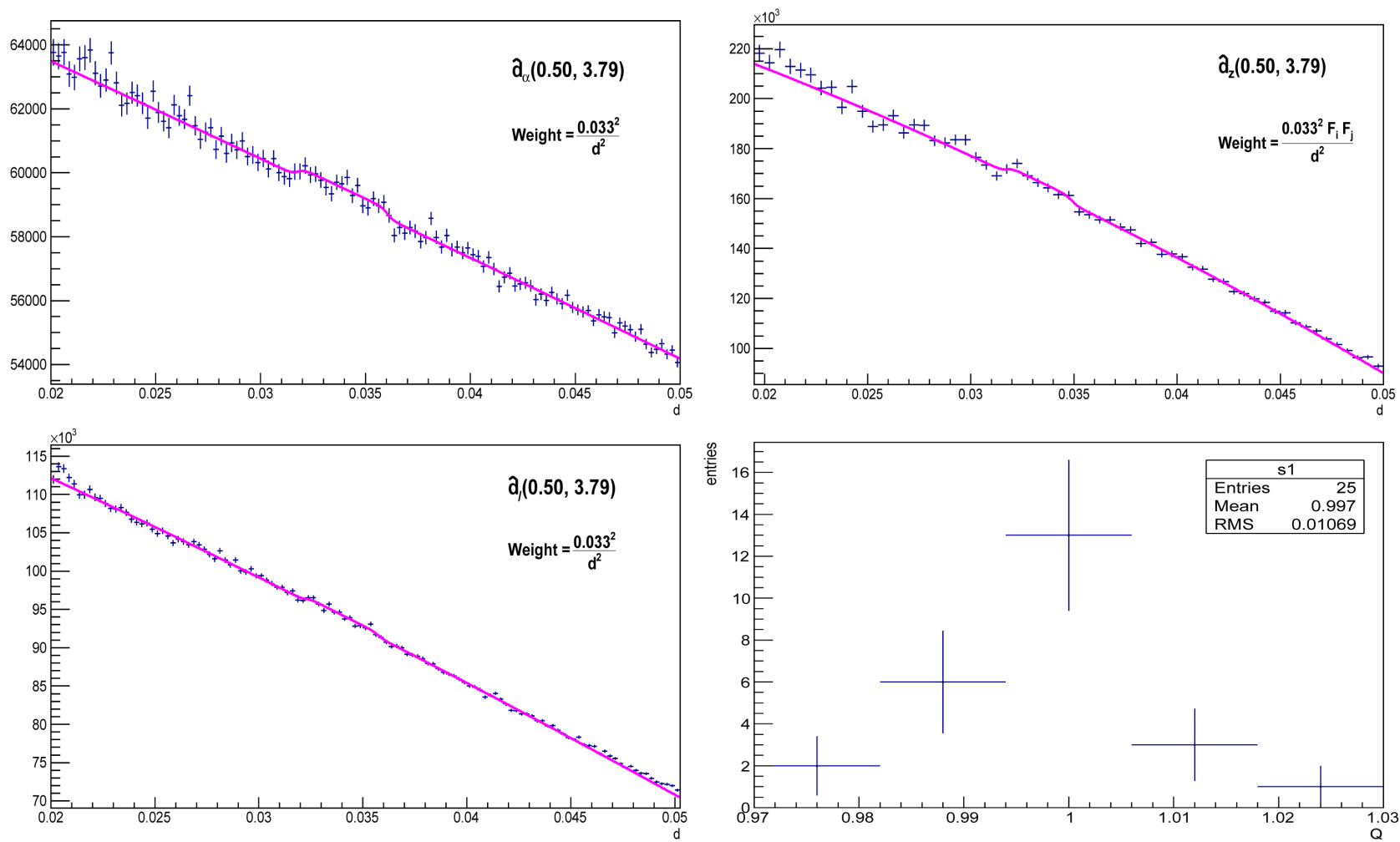

Figure 1. Fits to histograms of $d(0.5,3.79)$ that obtain the BAO distances $\hat{d}_{\alpha}(0.5,3.79), \hat{d}_{z}(0.5,3.79)$, and $\hat{d}_{/}(0.5,3.79)$ in the northern galactic cap, and distribution of the consistency parameter $Q$ for the $25 N$ or $S$ successful runs.

The fitting function is a second degree polynomial for the background and, for the BAO signal, a step-up-step-down function of the form

$$
\frac{\exp \left(x_{<}\right)}{\exp \left(x_{<}\right)+\exp \left(-x_{<}\right)}-\frac{\exp \left(x_{>}\right)}{\exp \left(x_{>}\right)+\exp \left(-x_{>}\right)}
$$

where

$$
x_{<}=\frac{d-\hat{d}+\Delta d}{\sigma}, \quad x_{>}=\frac{d-\hat{d}-\Delta d}{\sigma} .
$$

A run is defined as "successful" if the fits to all three histograms converge with a signal-to-background ratio significance greater than 1 standard deviation (raising this cut further obtains little improvement due to the cosmological fluctuations of the background), and the consistency parameter $Q$ is in the range 0.97 to 1.03 (if $Q$ is outside of this range then at least one of the fits has converged on a fluctuation of the background instead of the BAO signal). We obtain 13 successful runs for $\mathrm{N}$ and 12 successful runs for $\mathrm{S}$ which are presented in Table 1 and Table 2 respectively. The histogram of the consistency parameter $Q$ for these 25 runs is presented in Figure 1.

For each bin of redshift $Z$ we select from Table 1 and Table 2 the run with least $|Q-1|$ and obtain the 18 independent BAO distances listed in Table 3. This Table 3 is the main result of the present analysis, and supersedes the corresponding tables for DR12 in References [8] and [12]. 
Table 1. Measured BAO distances $\hat{d}_{\alpha}\left(z, z_{c}\right), \hat{d}_{z}\left(z, z_{c}\right)$, and $\hat{d}_{/}\left(z, z_{c}\right)$ in units of $c / H_{0}$ with $z_{c}=3.79$ (see text) from SDSS DR13 galaxies with right ascension $110^{\circ}$ to $270^{\circ}$, and declination $-5^{0}$ to $70^{\circ}$ in the northern galactic cap, i.e. dec $>27.0^{\circ}-17.0^{\circ}\left[\left(\mathrm{ra}-185.0^{\circ}\right) /\left(260.0^{\circ}-185.0^{\circ}\right)\right]^{2}$. Uncertainties are statistical from the fits to the BAO signal. Each BAO distance has an independent total uncertainty 0.00055 for $\hat{d}_{\alpha}\left(z, z_{c}\right)$ and $\hat{d}_{/}\left(z, z_{c}\right)$, or 0.00093 for $\hat{d}_{z}\left(z, z_{c}\right)$. No corrections have been applied.

\begin{tabular}{cccccccccc}
\hline$z$ & $z_{\min }$ & $z_{\max }$ & Galaxies & Centers & Type & $100 \hat{d}_{\alpha}\left(z, z_{c}\right)$ & $100 \hat{d}_{z}\left(z, z_{c}\right)$ & $100 \hat{d}_{(}\left(z, z_{c}\right)$ & $Q$ \\
\hline 0.14 & 0.10 & 0.20 & 152,785 & 3729 & G-C & $3.367 \pm 0.014$ & $3.284 \pm 0.014$ & $3.306 \pm 0.018$ & 0.993 \\
0.14 & 0.10 & 0.20 & 152,785 & 2853 & G-C & $3.376 \pm 0.017$ & $3.375 \pm 0.010$ & $3.363 \pm 0.017$ & 0.996 \\
0.25 & 0.20 & 0.30 & 58,670 & 3271 & G-C & $3.271 \pm 0.019$ & $3.499 \pm 0.020$ & $3.414 \pm 0.020$ & 1.014 \\
0.31 & 0.25 & 0.35 & 69,271 & 3677 & G-C & $3.344 \pm 0.025$ & $3.521 \pm 0.017$ & $3.409 \pm 0.016$ & 0.997 \\
0.35 & 0.30 & 0.40 & 83,515 & 58,491 & G-LG & $3.354 \pm 0.013$ & $3.320 \pm 0.010$ & $3.350 \pm 0.012$ & 1.003 \\
0.40 & 0.35 & 0.45 & 91,672 & 4180 & G-C & $3.479 \pm 0.025$ & $3.358 \pm 0.028$ & $3.380 \pm 0.025$ & 0.987 \\
0.46 & 0.40 & 0.50 & 137,972 & 137,972 & G-G & $3.523 \pm 0.011$ & $3.420 \pm 0.019$ & $3.485 \pm 0.007$ & 1.002 \\
0.46 & 0.40 & 0.50 & 137,972 & 55,925 & G-LG & $3.450 \pm 0.014$ & $3.423 \pm 0.011$ & $3.456 \pm 0.007$ & 1.005 \\
0.50 & 0.45 & 0.55 & 195,144 & 1514 & G-C & $3.436 \pm 0.024$ & $3.285 \pm 0.015$ & $3.431 \pm 0.012$ & 1.018 \\
0.50 & 0.45 & 0.55 & 195,144 & 5935 & G-C & $3.391 \pm 0.019$ & $3.332 \pm 0.020$ & $3.401 \pm 0.014$ & 1.011 \\
0.55 & 0.50 & 0.60 & 188,410 & 1105 & G-C & $3.338 \pm 0.021$ & $3.293 \pm 0.018$ & $3.300 \pm 0.026$ & 0.971 \\
0.64 & 0.60 & 0.70 & 81,624 & 81,624 & G-G & $3.368 \pm 0.014$ & $3.572 \pm 0.021$ & $3.439 \pm 0.012$ & 0.995 \\
0.64 & 0.60 & 0.70 & 81,624 & 33,982 & G-LG & $3.378 \pm 0.009$ & $3.586 \pm 0.016$ & $3.481 \pm 0.009$ & 1.004 \\
\hline
\end{tabular}

Table 2. Measured BAO distances $\hat{d}_{\alpha}\left(z, z_{c}\right), \hat{d}_{z}\left(z, z_{c}\right)$, and $\hat{d}_{/}\left(z, z_{c}\right)$ in units of $c / H_{0}$ with $z_{c}=3.79$ (see text) from SDSS DR13 galaxies with right ascension $110^{\circ}$ to $270^{\circ}$, and declination $-5^{0}$ to $70^{\circ}$ in the southern galactic cap, i.e. dec $<27.0^{\circ}-17.0^{\circ}\left[\left(\mathrm{ra}-185.0^{\circ}\right) /\left(260.0^{\circ}-185.0^{\circ}\right)\right]^{2}$. Uncertainties are statistical from the fits to the BAO signal. Each BAO distance has an independent total uncertainty 0.00055 for $\hat{d}_{\alpha}\left(z, z_{c}\right)$ and $\hat{d}_{l}\left(z, z_{c}\right)$, or 0.00093 for $\hat{d}_{z}\left(z, z_{c}\right)$. No corrections have been applied.

\begin{tabular}{cccccccccc}
\hline$Z$ & $Z_{\min }$ & $Z_{\max }$ & Galaxies & Centers & Type & $100 \hat{d}_{\alpha}\left(z, z_{c}\right)$ & $100 \hat{d}_{z}\left(z, z_{c}\right)$ & $100 \hat{d}_{(}\left(z, z_{c}\right)$ & $Q$ \\
\hline 0.19 & 0.15 & 0.25 & 58,381 & 1538 & G-C & $3.338 \pm 0.024$ & $3.315 \pm 0.037$ & $3.321 \pm 0.011$ & 0.998 \\
0.25 & 0.20 & 0.30 & 38,931 & 3865 & G-C & $3.311 \pm 0.035$ & $3.381 \pm 0.016$ & $3.360 \pm 0.013$ & 1.006 \\
0.31 & 0.25 & 0.35 & 46,916 & 2828 & G-C & $3.277 \pm 0.017$ & $3.438 \pm 0.027$ & $3.333 \pm 0.018$ & 0.996 \\
0.31 & 0.25 & 0.35 & 46,916 & 2559 & G-C & $3.271 \pm 0.028$ & $3.309 \pm 0.042$ & $3.295 \pm 0.036$ & 1.002 \\
0.46 & 0.40 & 0.50 & 91,599 & 91,599 & G-G & $3.319 \pm 0.013$ & $3.509 \pm 0.022$ & $3.366 \pm 0.016$ & 0.990 \\
0.46 & 0.40 & 0.50 & 91,599 & 37,456 & G-LG & $3.323 \pm 0.019$ & $3.445 \pm 0.024$ & $3.369 \pm 0.016$ & 0.998 \\
0.46 & 0.40 & 0.50 & 37,456 & 37,456 & LG-LG & $3.390 \pm 0.019$ & $3.536 \pm 0.013$ & $3.367 \pm 0.014$ & 0.975 \\
0.64 & 0.60 & 0.70 & 53,518 & 53,518 & G-G & $3.424 \pm 0.015$ & $3.327 \pm 0.019$ & $3.373 \pm 0.016$ & 0.997 \\
0.64 & 0.60 & 0.70 & 53,518 & 23,384 & G-LG & $3.393 \pm 0.017$ & $3.427 \pm 0.028$ & $3.373 \pm 0.019$ & 0.990 \\
0.64 & 0.60 & 0.70 & 53,518 & 941 & G-C & $3.349 \pm 0.014$ & $3.316 \pm 0.046$ & $3.346 \pm 0.020$ & 1.003 \\
0.64 & 0.60 & 0.70 & 23,384 & 23,384 & LG-LG & $3.446 \pm 0.016$ & $3.371 \pm 0.026$ & $3.381 \pm 0.015$ & 0.991 \\
0.64 & 0.60 & 0.70 & 53,518 & 689 & G-C & $3.416 \pm 0.020$ & $3.317 \pm 0.021$ & $3.316 \pm 0.036$ & 0.983 \\
\hline
\end{tabular}

\section{Uncertainties}

Histograms of BAO distances $d\left(z, z_{c}\right)$ have statistical fluctuations, and fluctuations of the background due to the clustering of galaxies as seen in Figure 1. These two types of fluctuations are the dominant source of the total uncertainties of the BAO distance measurements. These uncertainties are independent for 
Table 3. Independent measured BAO distances $\hat{d}_{\alpha}\left(z, z_{c}\right), \hat{d}_{z}\left(z, z_{c}\right)$, and $\hat{d}_{/}\left(z, z_{c}\right)$ in units of $c / H_{0}$ with $z_{c}=3.79$ (see text) obtained by selecting, for each bin of $z$, the entry with least $|Q-1|$ in Table 1 or Table 2. Each BAO distance has an independent total uncertainty 0.00030 for $\hat{d}_{\alpha}\left(z, z_{c}\right)$ and $\hat{d}_{/}\left(z, z_{c}\right)$, or 0.00060 for $\hat{d}_{z}\left(z, z_{c}\right)$. No corrections have been applied.

\begin{tabular}{cccccc}
\hline$z$ & $z_{\min }$ & $z_{\max }$ & $100 \hat{d}_{\alpha}\left(z, z_{c}\right)$ & $100 \hat{d}_{z}\left(z, z_{c}\right)$ & $100 \hat{d}_{(}\left(z, z_{c}\right)$ \\
\hline 0.14 & 0.1 & 0.2 & 3.376 & 3.375 & 3.363 \\
0.25 & 0.2 & 0.3 & 3.311 & 3.381 & 3.360 \\
0.35 & 0.3 & 0.4 & 3.354 & 3.320 & 3.350 \\
0.46 & 0.4 & 0.5 & 3.323 & 3.445 & 3.369 \\
0.55 & 0.5 & 0.6 & 3.338 & 3.293 & 3.300 \\
0.64 & 0.6 & 0.7 & 3.349 & 3.316 & 3.346 \\
\hline
\end{tabular}

each entry in Table 3. We present several estimates of the total uncertainties of the entries in Tables 1-3 extracted directly from the fluctuations of the numbers in these tables. All uncertainties in this article are at $68 \%$ confidence level.

We neglect the variation of $\hat{d}_{\alpha}\left(z, z_{c}\right), \hat{d}_{z}\left(z, z_{c}\right)$, and $\hat{d}_{/}\left(z, z_{c}\right)$ between adjacent bins of $z$ with respect to their uncertainties. The root-mean-square (r.m.s.) differences divided by $\sqrt{2}$ between corresponding rows in Table 1 and Table 2 for $\hat{d}_{\alpha}\left(z, z_{c}\right), \hat{d}_{z}\left(z, z_{c}\right)$, and $\hat{d}_{l}\left(z, z_{c}\right)$ are $0.00055,0.00093$, and 0.00054 respectively. We assign these numbers as total uncertainties of each entry in Table 1 and Table 2.

The 18 entries in Table 3 are independent. The r.m.s. differences for rows 1 $2,3-4$ and $5-6$ divided by $\sqrt{2}$ are $0.00030,0.00052$, and 0.00020 for $\hat{d}_{\alpha}\left(z, z_{c}\right), \hat{d}_{z}\left(z, z_{c}\right)$, and $\hat{d}_{/}\left(z, z_{c}\right)$ respectively.

The average and standard deviation of the columns $\hat{d}_{\alpha}\left(z, z_{c}\right), \hat{d}_{z}\left(z, z_{c}\right)$, and $\hat{d}_{/}\left(z, z_{c}\right)$ in Table 3 are respectively $0.03342,0.00021 ; 0.03355,0.00051$; and $0.03348,0.00023$.

The r.m.s. of $(1-Q)$ for Table 1 and Table 2 is 0.0111 . The average of all entries in Table 1 and Table 2 is 0.03383 . From the above estimates we take the uncertainties of $\hat{d}_{\alpha}\left(z, z_{c}\right), \hat{d}_{z}\left(z, z_{c}\right)$, and $\hat{d}_{/}\left(z, z_{c}\right)$ to be in the ratio $1: 2: 1$. From these numbers, we calculate the independent total uncertainties of $\hat{d}_{\alpha}\left(z, z_{c}\right)$, $\hat{d}_{z}\left(z, z_{c}\right)$, and $\hat{d}_{l}\left(z, z_{c}\right)$ to be $0.00026,0.00052$, and 0.00026 respectively.

From these estimates, we take the following independent total uncertainties for each entry of $\hat{d}_{\alpha}\left(z, z_{c}\right), \hat{d}_{z}\left(z, z_{c}\right)$, and $\hat{d}_{/}\left(z, z_{c}\right)$ in Table 3: 0.00030, 0.00060 , and 0.00030 respectively.

\section{Corrections}

Let us consider corrections to the $\mathrm{BAO}$ distances due to peculiar velocities and peculiar displacements of galaxies towards their centers. A relative peculiar velocity $v_{p}$ towards the center causes a reduction of the BAO distances $\hat{d}_{\alpha}\left(z, z_{c}\right), \hat{d}_{z}\left(z, z_{c}\right)$, and $\hat{d}_{l}\left(z, z_{c}\right)$ of order $0.5 v_{p} / c$. In addition, the Doppler shift produces an apparent shortening of $\hat{d}_{z}\left(z, z_{c}\right)$ by $v_{p} / c$, and somewhat less for $\hat{d}_{/}\left(z, z_{c}\right)$. 
We multiply the measured BAO distances $\hat{d}_{\alpha}\left(z, z_{c}\right), \hat{d}_{z}\left(z, z_{c}\right)$, and $\hat{d}_{l}\left(z, z_{c}\right)$ by correction factors $f_{\alpha}, f_{z}$ and $f_{/}$respectively. Simulations in Reference [6] obtain $f_{\alpha}-1=0.2283 \pm 0.0609 \%$ and $f_{z}-1=0.2661 \pm 0.0820 \%$ at $z=0.3, f_{\alpha}-1=0.1286 \pm 0.0425 \%$ and $f_{z}-1=0.1585 \pm 0.0611 \%$ at $z=1$, and $f_{\alpha}-1=0.0435 \pm 0.0293 \%$ and $f_{z}-1=0.0582 \pm 0.0402 \%$ at $z=3$. In the following sections we present fits with the corrections

$$
f_{\alpha}-1=0.00320 \cdot a^{1.35}, \quad f_{z}-1=0.00381 \cdot a^{1.35}, \quad f_{/}-1=0.00350 \cdot a^{1.35} .
$$

The effect of these corrections can be seen by comparing the first two fits in Table 4 below. An order-of-magnitude estimate of this correction can be obtained by calculating the r.m.s. $v_{p}$ corresponding to modes with $k \equiv 2 \pi / \lambda<2 \pi /\left(4 d_{\mathrm{BAO}}^{\prime}\right)$ with Equation (11) of Reference [5] and normalizing the result to $\sigma_{8}$, i.e. to the r.m.s. density fluctuation in a volume $(8 \mathrm{Mpc} / h)^{3}$.

\section{Measurements of $\Omega_{k}$ and $\Omega_{\mathrm{DE}}(a)$ from Uncalibrated BAO}

We consider five scenarios:

1) The observed acceleration of the expansion of the universe is due to the cosmological constant, i.e. $\Omega_{\mathrm{DE}}(a)$ is constant.

2) The observed acceleration of the expansion of the universe is due to a gas of negative pressure with an equation of state $w \equiv p / \rho<0$. We allow the index $w$ be a function of $a$ [3] [17] [18]: $w(a)=w_{0}+w_{a}(1-a)$. While this gas dominates $E(a)$ Equation [2]

$$
\frac{\mathrm{d} \rho}{\mathrm{d} t}=-3 \frac{\mathrm{d} a / \mathrm{d} t}{a}(\rho+p)
$$

can be integrated with the result [3] [17] [18]

$$
\Omega_{\mathrm{DE}}(a)=\Omega_{\mathrm{DE}} a^{-3\left(1+w_{0}+w_{a}\right)} \exp \left\{-3 w_{a}(1-a)\right\} .
$$

If $w_{0}=-1$ and $w_{a}=0$ we obtain constant $\Omega_{\mathrm{DE}}(a)$ as in the General Theory of Relativity.

3) Same as Scenario 2 with $w(a)$ constant, i.e. $w_{a}=0$.

Table 4. Cosmological parameters obtained from the 18 independent BAO measurements in Table 3 in several scenarios. Corrections for peculiar motions are given by Equation (11) except, for comparison, the fit " 1 " which has no correction. Scenario 1 has $\Omega_{\mathrm{DE}}(a)$ constant. Scenario 3 has $w=w_{0}$. Scenario 4 has $\Omega_{\mathrm{DE}}(a)=\Omega_{\mathrm{DE}}\left[1+w_{1}(1-a)\right]$.

\begin{tabular}{ccccccc}
\hline & Scenario ${ }^{*}$ & Scenario 1 & Scenario 1 & Scenario 3 & Scenario 4 & Scenario 4 \\
\hline$\Omega_{k}$ & 0 fixed & 0 fixed & $0.173 \pm 0.173$ & 0 fixed & 0 fixed & $0.151 \pm 0.185$ \\
$\Omega_{\mathrm{DE}}+0.5 \Omega_{k}$ & $0.714 \pm 0.014$ & $0.716 \pm 0.014$ & $0.710 \pm 0.016$ & $0.772 \pm 0.094$ & $0.749 \pm 0.049$ & $0.732 \pm 0.052$ \\
$w_{0}$ & n.a. & n.a. & n.a. & $-0.84 \pm 0.22$ & n.a. & n.a. \\
$w_{1}$ & n.a. & n.a. & n.a. & n.a. & $0.44 \pm 0.60$ & $0.33 \pm 0.70$ \\
$100 d_{\mathrm{BAO}}$ & $3.38 \pm 0.02$ & $3.39 \pm 0.02$ & $3.38 \pm 0.03$ & $3.36 \pm 0.05$ & $3.36 \pm 0.05$ & $3.36 \pm 0.05$ \\
$\chi^{2} /$ d.f. & $10.9 / 16$ & $11.2 / 16$ & $10.2 / 15$ & $10.6 / 15$ & $10.7 / 15$ & $10.0 / 14$ \\
\hline
\end{tabular}


4) We assume $\Omega_{\mathrm{DE}}(a)=\Omega_{\mathrm{DE}}\left[1+w_{1}(1-a)\right]$.

5) $\Omega_{\mathrm{DE}}(a)$ is arbitrary and needs to be measured at every $a$.

Note that BAO measurements can constrain $\Omega_{\mathrm{DE}}(a)$ for $0.3 \lesssim a \leq 1$ where $\Omega_{\mathrm{DE}}(a)$ contributes significantly to $E(a)$.

Let us try to understand qualitatively how the BAO distance measurements presented in Table 3 constrain the cosmological parameters. In the limit $z \rightarrow 0$ we obtain $d_{\mathrm{BAO}}=\hat{d}_{\alpha}\left(0, z_{c}\right)=\hat{d}_{z}\left(0, z_{c}\right)=\hat{d}_{/}\left(0, z_{c}\right)$, so the first row with $z=0.14$ in Table 3 approximately determines $d_{\mathrm{BAO}}$. This $d_{\mathrm{BAO}}$ and the measurement of, for example, $\hat{d}_{z}\left(0.3, z_{c}\right)$ then constrains the derivative of $\Omega_{\mathrm{m}} / a^{3}+\Omega_{\mathrm{DE}}+\Omega_{k} / a^{2}$ with respect to $a$ at $\mathrm{z} \approx 0.3$, i.e. constrains approximately $\Omega_{\mathrm{DE}}+0.5 \Omega_{k}$. We need an additional constraint for Scenario $1 . d_{\mathrm{BAO}}$ and $\theta_{\mathrm{MC}}$ constrain the last two factors in Equation (10), i.e. approximately constrain $\Omega_{\mathrm{DE}}+2.1 \Omega_{k}$. The additional BAO distance measurements in Table 3 then also constrain $w_{0}$ and $w_{a}$, or $w_{1}$.

In Table 4, we present the cosmological parameters obtained by minimizing the $\chi^{2}$ with 18 terms corresponding to the 18 independent BAO distance measurements in Table 3 for several scenarios. We find that the data is in agreement with the simplest cosmology with $\Omega_{k}=0$ and $\Omega_{\mathrm{DE}}(a)$ constant with $\chi^{2}$ per degree of freedom (d.f.) $11.2 / 16$, so no additional parameter is needed to obtain a good fit to this data. For free $\Omega_{k}$ we obtain $\Omega_{\mathrm{DE}}+0.5 \Omega_{k}=0.710 \pm 0.016$ for constant $\Omega_{\mathrm{DE}}(a)$, or $0.732 \pm 0.052$ if $\Omega_{\mathrm{DE}}(a)$ is allowed to depend on $a$ as in Scenario 4 . We present the variable $\Omega_{\mathrm{DE}}+0.5 \Omega_{k}$ instead of $\Omega_{\mathrm{DE}}$ because it has a smaller uncertainty. The constraints on $\Omega_{k}$ are weak.

In Table 5 we present the cosmological parameters obtained by minimizing the $\chi^{2}$ with 19 terms corresponding to the 18 BAO distance measurements listed in Table 3 plus the measurement of the correlation angle $\theta_{\mathrm{MC}}$ of the CMB given in Equation (9). We present the variable $\Omega_{\mathrm{DE}}+2.2 \Omega_{k}$ instead of $\Omega_{\mathrm{DE}}$ because it has a smaller uncertainty. We obtain

$$
\Omega_{k}=-0.015 \pm 0.030, \Omega_{\mathrm{DE}}+2.2 \Omega_{k}=0.717 \pm 0.004, w_{1}=0.37 \pm 0.61,
$$

when $\Omega_{\mathrm{DE}}(a)$ is allowed to vary as in Scenario 4 . There is no tension between

Table 5. Cosmological parameters obtained from the $18 \mathrm{BAO}$ measurements in Table 3 plus $\theta_{\mathrm{MC}}$ from Equation (9) in several scenarios. Corrections for peculiar motions are given by Equation (11). Scenario 1 has $\Omega_{\mathrm{DE}}(a)$ constant. Scenario 2 has $w(a)=w_{0}+w_{a}(1-a)$. Scenario 3 has $w=w_{0}$. Scenario 4 has $\Omega_{\mathrm{DE}}(a)=\Omega_{\mathrm{DE}}\left[1+w_{1}(1-a)\right]$.

\begin{tabular}{ccccccc}
\hline & Scenario 1 & Scenario 1 & Scenario 2 & Scenario 3 & Scenario 4 & Scenario 4 \\
\hline$\Omega_{k}$ & 0 fixed & $0.002 \pm 0.007$ & 0 fixed & 0 fixed & 0 fixed & $-0.015 \pm 0.030$ \\
$\Omega_{\mathrm{DE}}+2.2 \Omega_{k}$ & $0.719 \pm 0.003$ & $0.718 \pm 0.004$ & $0.708 \pm 0.015$ & $0.718 \pm 0.004$ & $0.718 \pm 0.004$ & $0.717 \pm 0.004$ \\
$w_{0}$ & n.a. & n.a. & $-0.87 \pm 0.19$ & $-0.99 \pm 0.04$ & n.a. & n.a. \\
$w_{a}$ or $w_{1}$ & n.a. & n.a. & $-0.60 \pm 0.93$ & n.a. & $0.06 \pm 0.15$ & $0.37 \pm 0.61$ \\
$100 d_{\text {вAO }}$ & $3.40 \pm 0.02$ & $3.39 \pm 0.02$ & $3.36 \pm 0.06$ & $3.39 \pm 0.03$ & $3.39 \pm 0.03$ & $3.37 \pm 0.05$ \\
$\chi^{2} /$ d.f. & $11.2 / 17$ & $11.2 / 16$ & $10.7 / 15$ & $11.1 / 16$ & $11.1 / 16$ & $10.8 / 15$ \\
\hline
\end{tabular}


the data and the case $\Omega_{k}=0$ and constant $\Omega_{\mathrm{DE}}(a)$ : with these two constraints we obtain $\Omega_{\mathrm{DE}}=0.719 \pm 0.003$ with $\chi^{2} /$ d.f. $=11.2 / 17$.

We now add BAO measurements with SDSS BOSS DR11 data of quasar Ly $\alpha$ forest cross-correlation at $z=2.36$ [10] and Ly $\alpha$ forest autocorrelation at $z=2.34$ [11]. From the combination in Reference [11] we obtain

$$
\frac{\chi(2.34)\left(1+\Omega_{k} \chi^{2}(2.34) / 6\right)}{d_{\mathrm{BAO}}(1+2.34)}=10.95 \pm 0.36, \quad \frac{1}{E(z=2.34) d_{\mathrm{BAO}}}=9.14 \pm 0.20 \text {. }
$$

From the $18 \mathrm{BAO}$ plus $\theta_{\mathrm{MC}}$ plus 2 Ly $\alpha$ measurements, for free $\Omega_{k}$, and $\Omega_{\mathrm{DE}}(a)$ allowed to vary as in Scenario 4 , we obtain $\Omega_{k}=-0.013 \pm 0.009$, $\Omega_{\mathrm{DE}}+2.2 \Omega_{k}=0.717 \pm 0.004$, and $w_{1}=0.34 \pm 0.24$. The $\chi^{2} /$ d.f. is $17.6 / 17$. Note that the Ly $\alpha$ measurements reduce the uncertainties of $\Omega_{k}$ and $w_{1}$. Requiring $\Omega_{k}=0$ and $\Omega_{\mathrm{DE}}(a)$ constant raises the $\chi^{2} /$ d.f. to $19.7 / 19$, so we observe no tension between the data and these two requirements, and obtain $\Omega_{\mathrm{DE}}=0.719 \pm 0.003$.

\section{Detailed Measurement of $\Omega_{\mathrm{DE}}(a)$}

We obtain $\Omega_{\mathrm{DE}}(a)$ from the 6 independent measurements of $\hat{d}_{z}\left(z, z_{c}\right)$ in Table 3, and Equations (1) and (6) for the case $\Omega_{k}=0$. The values of $d_{\mathrm{BAO}}$ and $\Omega_{\mathrm{m}}=1-\Omega_{\mathrm{DE}}(1)-\Omega_{k}$ are obtained from the fit for Scenario 4 in Table 5 . The results are presented in Figure 2. To guide the eye, we also show the straight

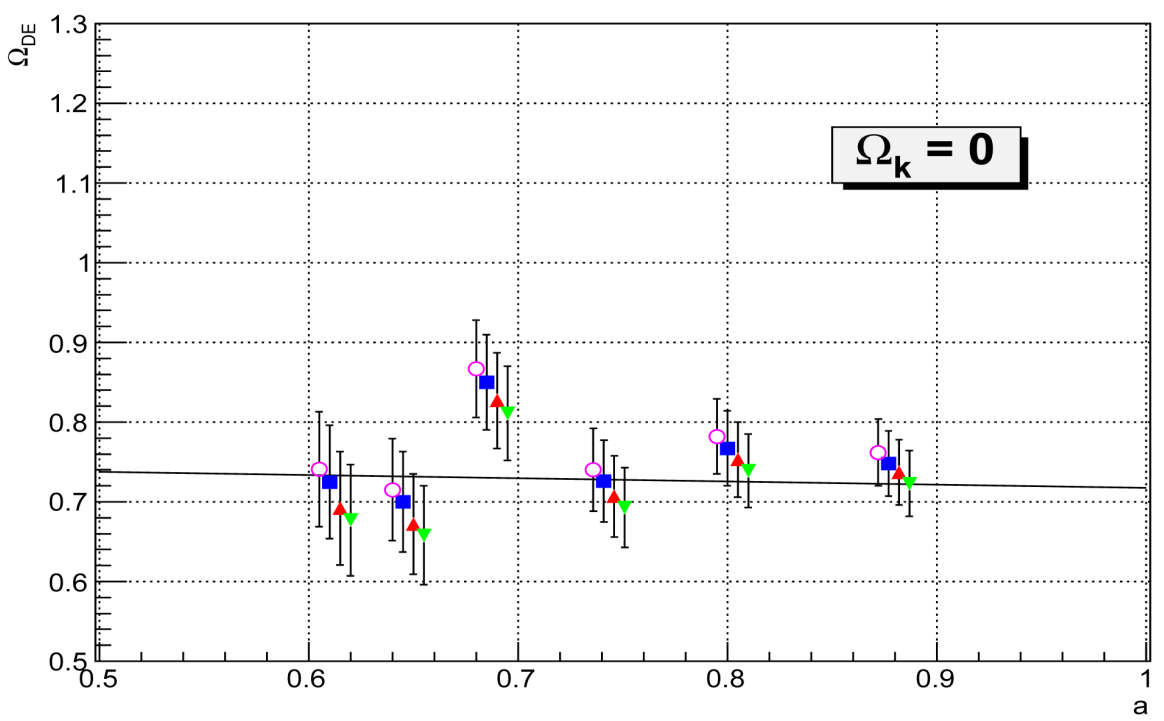

Figure 2. Measurements of $\Omega_{\mathrm{DE}}(a)$ obtained from the $6 \hat{d}_{z}\left(z, z_{c}\right)$ in Table 3 for $\Omega_{k}=0$, and the corresponding $d_{\mathrm{BAO}}$ and $\Omega_{\mathrm{DE}}$ from the fit for Scenario 4 in Table 5. The straight line is $\Omega_{\mathrm{DE}}(a)=0.718[1+0.055(1-a)]$ from the central values of this fit. The uncertainties correspond only to the total uncertainties of $\hat{d}_{z}\left(z, z_{c}\right)$. To illustrate correlated uncertainties we present results for $\left(d_{\mathrm{BAO}}, \Omega_{\mathrm{DE}}\right)=(0.0339,0.718+0.004)$ (squares), (0.0339,0.718-0.004) (triangles), $(0.0339+0.0003,0.718)$ (inverted triangles), and (0.0339-0.0003,0.718) (circles). For clarity some offsets in $a$ have been applied. 
line corresponding to the central values of $\Omega_{\mathrm{DE}}$ and $w_{1}$ of the fit for Scenario 4. In Figure 3 we present the results for offset bins of $z$ (which are partially correlated with the entries in Figure 2).

\section{Measurements of $\Omega_{k}, \Omega_{\mathrm{DE}}(a)$ and $N_{\mathrm{eq}}$ from Calibrated BAO}

Up to this point, we have used the BAO distance $d_{\mathrm{BAO}} f=r_{S}$ as an uncalibrated standard ruler. The cosmological parameters $h$ and $\Omega_{\mathrm{b}} h^{2}$ drop out of such an analysis, and the dependences of the results on $N_{\text {eq }}$ are not significant. $\Omega_{\mathrm{b}} \equiv \rho_{\mathrm{b} 0} / \rho_{\text {crit }}$ is the present density of baryons relative to the critical density. In this section we consider the $\mathrm{BAO}$ distance as a calibrated standard ruler to constrain the cosmological parameters $\Omega_{k}, \Omega_{\mathrm{DE}}(a), N_{\mathrm{eq}}, h$ and $\Omega_{\mathrm{b}} h^{2}$.

The sound horizon is calculated from first principles [1] as follows:

$$
r_{S}^{\prime}=\int_{0}^{t_{\mathrm{dec}}} \frac{C_{s} \mathrm{~d} t}{a}=\int_{0}^{a_{\mathrm{dec}}} \frac{c_{s} \mathrm{~d} a}{H_{0} a^{2} E(a)},
$$

where the speed of sound is

$$
c_{s}=\frac{c}{\sqrt{3\left(1+3 \rho_{\mathrm{b} 0} a /\left(4 \rho_{\gamma 0}\right)\right)}} .
$$

We can write the result for our purposes as

$$
r_{S}=0.03389 \times A \times\left(\frac{0.30}{\Omega_{\mathrm{m}}}\right)^{0.255},
$$

where

$$
A=\left(\frac{h}{0.72}\right)^{0.489}\left(\frac{0.023}{\Omega_{\mathrm{b}} h^{2}}\right)^{0.098}\left(\frac{3.36}{N_{\text {eq }}}\right)^{0.245}
$$

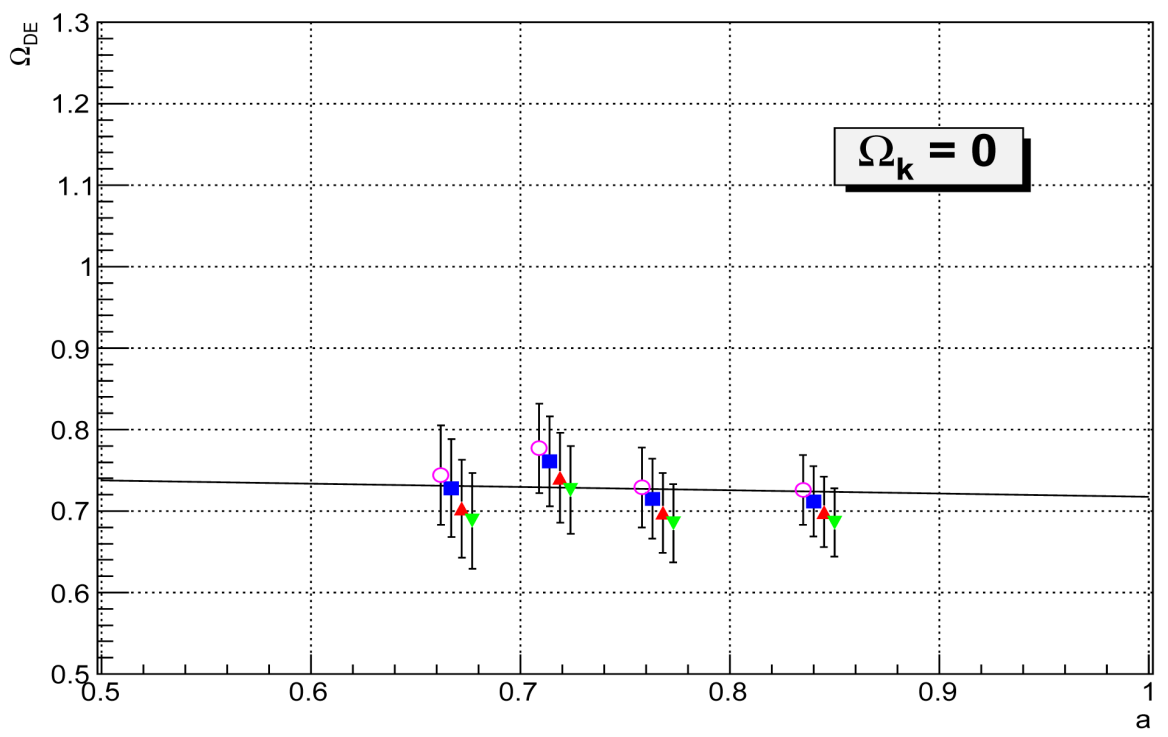

Figure 3. Same as Figure 2 for offset bins of $z$ with least $|Q-1|$ in Table 1 or Table 2. These measurements are partially correlated with those of Figure 2. 
(we have neglected the dependence of $z_{\mathrm{dec}}=1089.9 \pm 0.4$ [2] [9] on the cosmological parameters).

In this paragraph we take $N_{\text {eq }}=3.38$ corresponding to 3 flavors of neutrinos [2]. From Big-Bang nucleosynthesis, $\Omega_{\mathrm{b}} h^{2}=0.0225 \pm 0.0008$ (at $68 \%$ confidence) [2]. With the latest direct measurement $h=0.720 \pm 0.030$ by the Hubble Space Telescope Key Project [19] we obtain $A=1.000 \pm 0.021$. An alternative choice is the Planck "TT + lowP + lensing" analysis [2], that assumes $\Omega_{k}=0$ and a $\Lambda$ CDM cosmology, that obtains $\Omega_{\mathrm{b}} h^{2}=0.02226 \pm 0.00023$, $h=0.678 \pm 0.009$ and $A=0.972 \pm 0.007$. The cosmological parameters that minimize the $\chi^{2}$ with 22 terms (18 BAO measurements from Table 3 plus $\theta_{\mathrm{MC}}$ from Equation (9) plus 2 Ly $\alpha$ measurements from Equation (15) plus A) are presented in Table 6 . Note that the addition of the external constraint from A slightly reduces the uncertainties of $\Omega_{k}$ and $w_{1}$ if $N_{\text {eq }}=3.38$ is fixed. Note in Table 6 that the data is consistent with the constraints $\Omega_{k}=0$ and constant $\Omega_{\mathrm{DE}}(a)$ for both values of $A$.

In this paragraph we let $N_{\text {eq }}$ be free. We turn the problem around: from 18 BAO measurements from Table 3 plus $\theta_{\mathrm{MC}}$ from Equation (9) plus 2 Ly $\alpha$ measurements from Equation (15) we constrain $A$. The results are

$A=0.965 \pm 0.014$ for free $\Omega_{k}$ and $\Omega_{\mathrm{DE}}(a)$ allowed to vary as in Scenario 4, $A=0.983 \pm 0.005$ for $\Omega_{k}=0$ fixed and $\Omega_{\mathrm{DE}}(a)$ allowed to vary as in Scenario 4 , and $A=0.9855 \pm 0.0012$ for $\Omega_{k}=0$ fixed and constant $\Omega_{\mathrm{DE}}(a)$. For free $\Omega_{k}, \Omega_{\mathrm{DE}}(a)$ allowed to vary as in Scenario 4,

$\Omega_{\mathrm{b}} h^{2}=0.0225 \pm 0.0008$, and $h=0.720 \pm 0.030$ we obtain $N_{\text {eq }}=3.92 \pm 0.40$ corresponding to $N_{\text {eff }}=4.2 \pm 0.9$ neutrino flavors. For $\Omega_{k}=0$ fixed, constant $\Omega_{\mathrm{DE}}(a), \Omega_{\mathrm{b}} h^{2}=0.02226 \pm 0.00023$, and $h=0.678 \pm 0.009$ we obtain $N_{\text {eq }}=3.20 \pm 0.09$ corresponding to $N_{\text {eff }}=2.64 \pm 0.20$ neutrino flavors.

\section{Comparison with Previous Measurements}

Let us compare the results obtained with SDSS DR13 data with DR12 data. The $\chi^{2}$ between Table 3 and Table III of Reference [8] is 44.8 for 18 degrees of freedom. The $\chi^{2}$ between Table 3 and Table III of Reference [12] is 25.9 for 17 degrees of freedom. The disagreement in both cases is due to the same two entries in Table III of Reference [8] or Table III of Reference [12] with miss-fits

Table 6. Cosmological parameters obtained from the $18 \mathrm{BAO}$ measurements in Table 3 plus $\theta_{\mathrm{MC}}$ from Equation (9) plus 2 Ly $\alpha$ measurements in Equation (15) plus $A$ in several scenarios. Corrections for peculiar motions are given by Equation (11). $N_{\text {eq }}=3.38$. Scenario 1 has $\Omega_{\mathrm{DE}}(a)$ constant. Scenario 4 has $\Omega_{\mathrm{DE}}(a)=\Omega_{\mathrm{DE}}\left[1+w_{1}(1-a)\right]$.

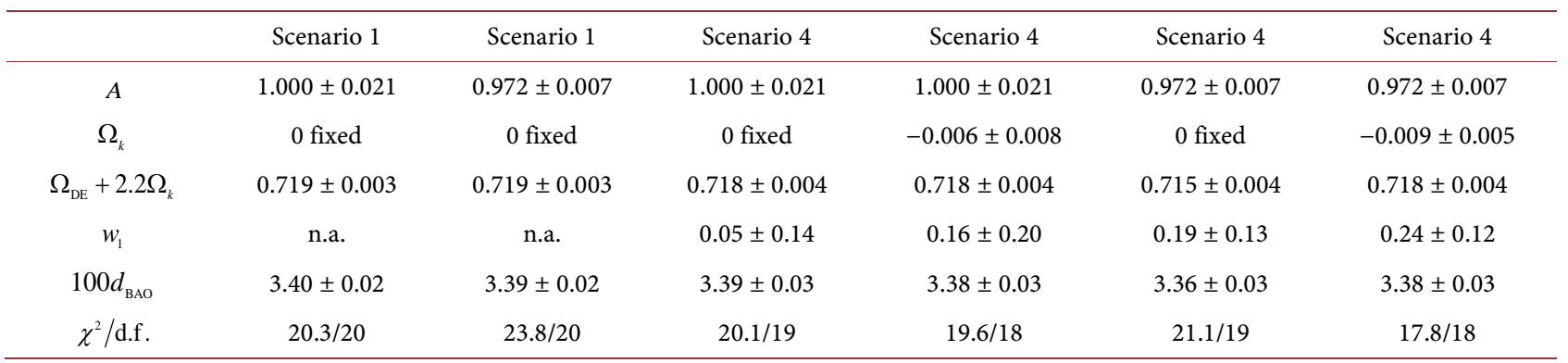


converging on background fluctuations instead of the $\mathrm{BAO}$ signal:

$\hat{d}_{\alpha}(0.46,3.79)$ and $\hat{d}_{/}(0.54,3.79)$. The fluctuation of $\hat{d}_{\alpha}(0.46,3.79)$ can be seen in Table 1 for the northern galactic cap, but not in Table 2 for the southern galactic cap. Removing the two miss-fits from the comparisons obtains $\chi^{2} /$ d.f. $=21.2 / 16$ and 11.1/15 respectively.

We compare Equation (14) for DR13 data, with the corresponding fits for DR12 data. From Table VIII of Reference [8]:

$$
\Omega_{k}=0.043 \pm 0.041, \quad \Omega_{\mathrm{DE}}+2.0 \Omega_{k}=0.716 \pm 0.006, \quad w_{1}=-0.16 \pm 0.94 .
$$

From Table VII of Reference [12]:

$$
\Omega_{k}=0.060 \pm 0.052, \quad \Omega_{\mathrm{DE}}+2.0 \Omega_{k}=0.717 \pm 0.007, \quad w_{1}=-0.86 \pm 1.26 .
$$

Note in Equation (14) how the DR13 data has lowered the uncertainties.

The final consensus measurements of the SDSS-III Baryon Oscillation Spectroscopic Survey [20] (an analysis of the DR12 galaxy sample), are presented in Table 7 (reproduced from Reference [12] for completness). There is agreement with the measurements of DR13 data in Table 3. The notation of Reference [20] is related to the notation of the present article as follows:

$$
\begin{gathered}
D_{M} \frac{r_{\mathrm{d}}, \text { fid }}{\mathrm{r}_{\mathrm{d}}}=\frac{c}{H_{0}} \chi(z)\left[1+\frac{1}{6} \Omega_{k} \chi^{2}(z)\right] \frac{r_{\mathrm{d}}, \text { fid }}{d_{\text {BAO }}^{\prime}}=r_{\mathrm{d}}, \text { fid } \frac{z \exp \left(-z / z_{c}\right)}{\hat{d}_{\alpha}\left(z, z_{c}\right)}, \\
H \frac{r_{\mathrm{d}}}{r_{\mathrm{d}}, \text { fid }}=H_{0} E(z) \frac{d_{\mathrm{BAO}}^{\prime}}{r_{\mathrm{d}}, \text { fid }}=\frac{c}{r_{\mathrm{d}}, \text { fid }} \frac{\hat{d}_{z}\left(z, z_{c}\right)}{\left(1-z / z_{c}\right) \exp \left(-z / z_{c}\right)},
\end{gathered}
$$

where $r_{\mathrm{d}}$, fid $=147.78 \mathrm{Mpc}$ and $H_{0}=67.6 \mathrm{~km} \cdot \mathrm{s}^{-1} \cdot \mathrm{Mpc}^{-1}$.

\section{Conclusions}

1) The main results of these studies are the independent measured $B A O$ observables $\hat{d}_{\alpha}\left(z, z_{c}\right), \hat{d}_{z}\left(z, z_{c}\right)$, and $\hat{d}_{l}\left(z, z_{c}\right)$ presented in Table 3 . It is difficult to distinguish the BAO signal from fluctuations of the background. To gain confidence in the results we have repeated the measurements many times with different galaxy selections to obtain different background fluctuations. Requiring successful fits for each of the three independent observables, for each bin of $z$, allows the use of the consistency relation (8) to discriminate against miss-fits on background fluctuations instead of the BAO signal. The consistency parameter $Q$ also allows quality control of the measurements, see Figure 1 . Table 3 for DR13 data supersedes the corresponding tables in References [8] and

Table 7. Final consensus "BAO + FS" measurements of the SDSS DR12 data set [20] (uncertainties are statistical and systematic), and the corresponding BAO parameters $\hat{d}_{\alpha}\left(z, z_{c}\right)$ and $\hat{d}_{z}\left(z, z_{c}\right)$ with $z_{c}=3.79$. These measurements include the peculiar motion corrections.

\begin{tabular}{ccccc}
\hline$z$ & $D_{M} r_{d}, \mathrm{fid} / r_{d}[\mathrm{Mpc}]$ & $100 \hat{d}_{\alpha}\left(z, z_{c}\right)$ & $H r_{d} / r_{d}, \operatorname{fid}\left[\mathrm{km} \cdot \mathrm{s}^{-1} \cdot \mathrm{Mpc}^{-1}\right]$ & $100 \hat{d}_{z}\left(z, z_{c}\right)$ \\
\hline 0.38 & $1518 \pm 20 \pm 11$ & $3.346 \pm 0.050$ & $81.5 \pm 1.7 \pm 0.9$ & $3.270 \pm 0.077$ \\
0.51 & $1977 \pm 23 \pm 14$ & $3.332 \pm 0.045$ & $90.5 \pm 1.7 \pm 1.0$ & $3.375 \pm 0.074$ \\
0.61 & $2283 \pm 28 \pm 16$ & $3.362 \pm 0.047$ & $97.3 \pm 1.8 \pm 1.1$ & $3.426 \pm 0.074$ \\
\hline
\end{tabular}


[12] for DR12 data.

2) From the $18 \mathrm{BAO}$ measurements in Table 3 , and no other input, we obtain

$$
\Omega_{k}=0.151 \pm 0.185, \Omega_{\mathrm{DE}}+0.5 \Omega_{k}=0.732 \pm 0.052, w_{1}=0.33 \pm 0.70,
$$

for $\Omega_{\mathrm{DE}}(a)$ allowed to vary as in Scenario 4. For $\Omega_{k}=0$ and constant $\Omega_{\mathrm{DE}}(a)$ we obtain $\Omega_{\mathrm{DE}}=0.716 \pm 0.014$, which may be compared to the independent Planck "TT + lowP + lensing" result (which assumes a $\Lambda \mathrm{CDM}$ cosmology with $\Omega_{k}=0$ ): $\Omega_{\mathrm{DE}}=0.692 \pm 0.012$ [2]. Note that these two results are based on independent cosmological measurements. See Table 4 for fits in several scenarios.

3) From $18 \mathrm{BAO}$ measurements plus $\theta_{\mathrm{MC}}$ from the $\mathrm{CMB}$ we obtain

$$
\Omega_{k}=-0.015 \pm 0.030, \Omega_{\mathrm{DE}}+2.2 \Omega_{k}=0.717 \pm 0.004, w_{1}=0.37 \pm 0.61,
$$

for $\Omega_{\mathrm{DE}}(a)$ allowed to vary as in Scenario 4. See Table 5 for fits in several scenarios. The cosmological parameters $h, \Omega_{\mathrm{b}} h^{2}$ and $N_{\mathrm{eq}}$ drop out of this analysis. Imposing the constraints $\Omega_{k}=0$ and constant $\Omega_{\mathrm{DE}}(a)$ obtains $\Omega_{\mathrm{DE}}=0.719 \pm 0.003$.

4) Detailed measurements of $\Omega_{\mathrm{DE}}(a)$ are presented in Figure 2 and Figure 3.

5) From $18 \mathrm{BAO}$ plus $\theta_{\mathrm{MC}}$ plus $2 \mathrm{Ly} \alpha$ measurements we obtain

$$
\begin{aligned}
& \Omega_{k}=-0.013 \pm 0.009, \Omega_{\mathrm{DE}}+2.2 \Omega_{k}=0.717 \pm 0.004, \\
& w_{1}=0.34 \pm 0.24, A=0.965 \pm 0.014,
\end{aligned}
$$

when $\Omega_{\mathrm{DE}}(a)$ is allowed to vary as in Scenario 4 . Note the constraint on $A$ defined in Equation (19). The corresponding constraint on $N_{\text {eq }}$ for $\Omega_{b} h^{2}=0.0225 \pm 0.0008$, and $h=0.720 \pm 0.030$ is $N_{\text {eq }}=3.92 \pm 0.40$ corresponding to $N_{\text {eff }}=4.2 \pm 0.9$ neutrino flavors.

For $\Omega_{k}=0$ and constant $\Omega_{\mathrm{DE}}(a)$ we obtain $A=0.9855 \pm 0.0012$. The corresponding constraint on $N_{\text {eq }}$ for $\Omega_{b} h^{2}=0.02226 \pm 0.00023$, and $h=0.678 \pm 0.009$ is $N_{\text {eq }}=3.20 \pm 0.09$ corresponding to $N_{\text {eff }}=2.64 \pm 0.20$ neutrino flavors.

6) From $18 \mathrm{BAO}$ plus $\theta_{\mathrm{MC}}$ plus $2 \mathrm{Ly} \alpha$ plus $A$ measurements with $N_{\text {eq }}=3.38$ fixed we obtain the results shown in Table 6. For $\Omega_{\mathrm{DE}}(a)$ allowed to vary as in Scenario 4 and $A=1.000 \pm 0.021$ we obtain

$\Omega_{k}=-0.006 \pm 0.008, \quad \Omega_{\mathrm{DE}}+2.2 \Omega_{k}=0.718 \pm 0.004, \quad w_{1}=0.16 \pm 0.20 .(27)$

7) For all data sets, we obtain no tension with the constraints $\Omega_{k}=0$ and constant $\Omega_{\mathrm{DE}}(a)$.

The SDSS has brought the measurements of $\Omega_{\mathrm{DE}}(a)$ with free $\Omega_{k}$ to a new level of precision.

\section{Acknowledgements}

Funding for the Sloan Digital Sky Survey IV has been provided by the Alfred P. Sloan Foundation, the US Department of Energy Office of Science, and the Participating Institutions. SDSS-IV acknowledges support and resources from the Center for High-Performance Computing at the University of Utah. The SDSS web site is www.sdss.org. 
SDSS-IV is managed by the Astrophysical Research Consortium for the Participating Institutions of the SDSS Collaboration including the Brazilian Participation Group, the Carnegie Institution for Science, Carnegie Mellon University, the Chilean Participation Group, the French Participation Group, HarvardSmithsonian Center for Astrophysics, Instituto de Astrofísica de Canarias, The Johns Hopkins University, Kavli Institute for the Physics and Mathematics of the Universe (IPMU)/University of Tokyo, Lawrence Berkeley National Laboratory, Leibniz Institut für Astrophysik Potsdam (AIP), Max-Planck-Institut für Astronomie (MPIA Heidelberg), Max-Planck-Institut für Astrophysik (MPA Garching), Max-Planck-Institut für Extraterrestrische Physik (MPE), National Astronomical Observatories of China, New Mexico State University, New York University, University of Notre Dame, Observatário Nacional/MCTI, The Ohio State University, Pennsylvania State University, Shanghai Astronomical Observatory, United Kingdom Participation Group, Universidad Nacional Autónoma de México, University of Arizona, University of Colorado Boulder, University of Oxford, University of Portsmouth, University of Utah, University of Virginia, University of Washington, University of Wisconsin, Vanderbilt University, and Yale University.

\section{References}

[1] Eisenstein, D.J., Seo, H.-J. and White, M. (2007) On the Robustness of the Acoustic Scale in the Low-Redshift Clustering of Matter. The Astrophysical Journal, 664, 660-674. https://doi.org/10.1086/518755

[2] Patrignani, C., et al. (2016) Review of Particle Physics. Chinese Physics C, 40, Article ID: 100001. https://doi.org/10.1088/1674-1137/40/10/100001

[3] Bruce, A. (2009) Bassett and Renée Hlozek. arXiv:0910.5224

[4] Weinberg, D.H., et al. (2013) Observational Probes of Cosmic Acceleration. arXiv:1201.2434

[5] Hoeneisen, B. (2000) A Simple Model of the Hierarchical Formation of Galaxies. arXiv:astro-ph/0009071

[6] Seo, H.-J., et al. (2010) High-Precision Predictions for the Acoustic Scale in the Nonlinear Regime. The Astrophysical Journal, 720, 1650.

https://doi.org/10.1088/0004-637X/720/2/1650

[7] Albareti, F.D., et al. (2016) SDSS Collaboration. arXiv:1608.02013

[8] Hoeneisen, B. (2016) Study of Baryon Acoustic Oscillations with SDSS DR12 Data and Measurement of $\Omega_{\mathrm{DE}}($ a). arXiv:1607.02424

[9] Planck Collaboration (2015) Results XIII, Astron. \& Astrophys. Submitted. arXiv:1502.01589v2

[10] Font-Ribera, A., et al. (2014) Quasar-Lyman $\alpha$ Forest Cross-Correlation from BOSS DR11: Baryon Acoustic Oscillations. arXiv:1311.1767

[11] Delubac, T., et al. (2014) Baryon Acoustic Oscillations in the Ly $\alpha$ Forest of BOSS DR11 Quasars. arXiv:1404.1801v2

[12] Hoeneisen, B. (2016) Study of Baryon Acoustic Oscillations with SDSS DR12 Data and Measurements of $\Omega_{k}$ and $\Omega_{\mathrm{DE}}($ a). Part II. arXiv:1608.08486

[13] Alam, S., et al. (2015) The Eleventh and Twelfth Data Releases of the Sloan Digital Sky Survey: Final Data from SDSS-III. Astrophysical Journal Supplement Series, 
219, 12. arXiv: 1501.00963

https://doi.org/10.1088/0067-0049/219/1/12

[14] Peacock, J.A. (1999) Cosmological Physics. Cambridge University Press, Cambridge.

[15] Dawson, K.S., Schlegel, D.J., Ahn, C.P., et al. (2013) The Baryon Oscillation Spectroscopic Survey of SDSS-III. Astronomical Journal, 145, 10.

https://doi.org/10.1088/0004-6256/145/1/10

[16] Dawson, K.S., Kneib, J.-P., Percival, W.J., et al. (2016) The SDSS-IV Extended Baryon Oscillation Spectroscopic Survey: Overview and Early Data. Astronomical Journal, 151, 44. https://doi.org/10.3847/0004-6256/151/2/44

[17] Chevallier, M. and Polarski, D. (2001) Accelerating Universes with Scaling Dark Matter. International Journal of Modern Physics D, 10, 213-223. https://doi.org/10.1142/S0218271801000822

[18] Linder, E.V. (2003) Exploring the Expansion History of the Universe. Physical Review Letters, 90, Article ID: 091301. https://doi.org/10.1103/PhysRevLett.90.091301

[19] Humphreys, E.M.L., Reid, M.J., Moran, J.M., Greenhill, L.J. and Argon, A.L. (2013) Toward a New Geometric Distance to the Active Galaxy NGC 4258. III. Final Results and the Hubble Constant. Astrophysical Journal, 775, 13. https://doi.org/10.1088/0004-637X/775/1/13

[20] Alam, S., et al. (2016) The Clustering of Galaxies in the Completed SDSS-III Baryon Oscillation Spectroscopic Survey: Cosmological Analysis of the DR12 Galaxy Sample. arXiv:1607.03155

Submit or recommend next manuscript to SCIRP and we will provide best service for you:

Accepting pre-submission inquiries through Email, Facebook, LinkedIn, Twitter, etc. A wide selection of journals (inclusive of 9 subjects, more than 200 journals)

Providing 24-hour high-quality service

User-friendly online submission system

Fair and swift peer-review system

Efficient typesetting and proofreading procedure

Display of the result of downloads and visits, as well as the number of cited articles

Maximum dissemination of your research work

Submit your manuscript at: http://papersubmission.scirp.org/

Or contact ijaa@scirp.org 